University of Nebraska - Lincoln

DigitalCommons@University of Nebraska - Lincoln

7-1-2005

\title{
Effects of electron irradiation on the ferroelectric properties of Langmuir-Blodgett copolymer films
}

Christina M. Othon

University of Nebraska-Lincoln, cothon@wesleyan.edu

F.B. Bateman

Ionizing Radiation Division, Physics Laboratory, National Institute of Standards and Technology, Gaithersburg, Maryland

Stephen Ducharme

University of Nebraska, sducharme1@unl.edu

Follow this and additional works at: https://digitalcommons.unl.edu/physicsducharme

Part of the Physics Commons

Othon, Christina M.; Bateman, F.B.; and Ducharme, Stephen, "Effects of electron irradiation on the ferroelectric properties of Langmuir-Blodgett copolymer films" (2005). Stephen Ducharme Publications. 4. https://digitalcommons.unl.edu/physicsducharme/4

This Article is brought to you for free and open access by the Research Papers in Physics and Astronomy at DigitalCommons@University of Nebraska - Lincoln. It has been accepted for inclusion in Stephen Ducharme Publications by an authorized administrator of DigitalCommons@University of Nebraska - Lincoln. 


\title{
Effects of electron irradiation on the ferroelectric properties of Langmuir-Blodgett copolymer films
}

\author{
C. M. Othon ${ }^{\text {a) }}$ \\ Department of Physics and Astronomy and the Center for Materials Research and Analysis, \\ University of Nebraska, Lincoln, Nebraska 68588-0111
}

\section{F. B. Bateman}

Ionizing Radiation Division, Physics Laboratory, National Institute of Standards and Technology, Gaithersburg, Maryland 20899-8460

\author{
Stephen Ducharme ${ }^{\text {b) }}$ \\ Department of Physics and Astronomy and the Center for Materials Research and Analysis, \\ University of Nebraska, Lincoln, Nebraska 68588-0111
}

(Received 22 October 2004; accepted 20 May 2005; published online 13 July 2005)

\begin{abstract}
The effect of irradiation on the ferroelectric properties of Langmuir-Blodgett films of the copolymer poly(vinylidene fluoride-trifluoroethylene) was investigated using 1-MeV electrons for doses from 0.16 to $1.10 \mathrm{MGy}$, where $1 \mathrm{Gray}(\mathrm{Gy})=100 \mathrm{rad}$. Irradiation causes a systematic decrease in the phase-transition temperature, crystallinity, and spontaneous polarization of the films. The crystallinity and spontaneous polarization of the films decreased by amounts proportional to the dose, both tending toward zero near a dose of $1.30 \mathrm{MGy}$. The ferroelectric-paraelectric phase-transition temperature, however, was only reduced by about $12 \%$, indicating that the primary effect of irradiation was to convert a crystalline ferroelectric material to a noncrystalline dielectric. () 2005 American Institute of Physics. [DOI: 10.1063/1.1954868]
\end{abstract}

\section{INTRODUCTION}

Polyvinylidene fluoride (PVDF) and its copolymers have long been studied for their striking ferroelectric properties and their applications in actuators, transducers, and ferroelectric memory. ${ }^{1}$ The homopolymer PVDF consists of a linear carbon-carbon chain with the repeating structure $-\left(\mathrm{CH}_{2}-\mathrm{CF}_{2}\right)^{-}$, which has a permanent dipole moment roughly perpendicular to the chain. The ferroelectric phase consists of a polar crystalline packing of chains with alltrans conformation and an estimated polarization of $0.13 \mathrm{C} / \mathrm{m}^{2}{ }^{2}$ Though PVDF does not crystallize well from the melt, polymorphous samples containing lamellar crystals and amorphous material can be fabricated by solvent-casting or spin-coating techniques. Mechanical, thermal, and electrical treatment of the films can produce samples of about 50\% crystallinity. ${ }^{1-3}$ Random copolymerization of PVDF with, e.g., $15 \%$ or more of trifluoroethylene (TrFE) suppresses the ferroelectric phase-transition temperature below the melting point, thus allowing samples to be annealed in the transgauche paraelectric phase, such that samples of $90 \%$ crystallinity or better are readily obtained. ${ }^{1-5}$ The addition of the larger, less polar TrFE monomer results in a slightly larger unit cell and reduces polarization to approximately $0.1 \mathrm{C} / \mathrm{m}^{2}{ }^{2}$ The ferroelectric phase transition for PVDF is above its melting temperature of $180^{\circ} \mathrm{C}$, while the $70: 30$ copolymer has a reduced phase-transition temperature in the range of $80-110^{\circ} \mathrm{C}$, well below its melting temperature of $150^{\circ} \mathrm{C}$, allowing a detailed study of the ferroelectricparaelectric phase transition. ${ }^{2}$

\footnotetext{
${ }^{a)}$ Electronic mail: cothon1@bigred.unl.edu

${ }^{b)}$ Electronic mail: sducharme1@unl.edu
}

It is well known that high-energy electron irradiation can have dramatic effects on the thermal, structural, and physical properties of PVDF and its copolymers. ${ }^{6-13}$ Electron irradiation lowers the melting point and the ferroelectricparaelectric phase-transition temperature by reducing the crystallite size, introducing defects, reducing the strain in crystallite regions, and decreasing the dipolar energy. ${ }^{6,7}$ $\mathrm{X}$-ray diffraction studies of vinylidene fluoride (VDF) copolymer samples show that irradiation with electrons of energy $1-3 \mathrm{MeV}$ and a dose of $0.6 \mathrm{MGy}(1 \mathrm{Gray}=100 \mathrm{rad})$ converts the ferroelectric crystals to a combination of an amorphous material and a phase that is structurally similar to the paraelectric phase. ${ }^{8,13}$ The original ferroelectric state of the irradiated samples usually could not be recovered, even by long anneals above the paraelectric phase transition. Electron irradiation of PVDF-TrFE copolymer films near the ferroelectric-paraelectric phase-transition temperature $\left(T_{C}\right)$ results in conversion to a ferroelectric relaxorlike state, characterized by a giant electrostriction $(\approx 4 \%-5 \%)$ with high elastic energy density. ${ }^{9,11}$ The electron irradiation also results in the reduction of polarization hysteresis. This reduction in hysteresis, which is desirable for electromechanical devices, cannot be recovered by application of large fields and is therefore indicative of the permanent structural change. It has been proposed that radiation-induced double bonds stabilize the paraelectric phase. ${ }^{7}$ Cheng et al. report three dose ranges of interest. ${ }^{11}$ Range I, for doses $<0.5 \mathrm{MGy}$, produces a mixture of polar and nonpolar phases. Range II, from 0.5 to $0.85 \mathrm{MGy}$, produces macroscopically uniform samples with little dielectric or thermal hysteresis, but a relaxorlike state indicating dipolar correlation even in nonferroelectric regions. Range III, above 1 MGy, produces a pre- 
dominantly amorphous matrix with some embedded crystallites. Similarly, proton irradiation has also been shown to produce a relaxorlike state in VDF copolymers. ${ }^{14}$

The focus of this study is on the effect of irradiation on the ferroelectric properties of ultrathin $(18-90 \mathrm{~nm}$ thick) films of the $\mathrm{P}(\mathrm{VDF}-\mathrm{TrFE})$ copolymer. These films were produced by Langmuir-Blodgett (LB) deposition using methods developed by Palto et al. in $1995 .{ }^{15}$ The quality of these films is excellent, with high crystallinity and crystalline orientation, ${ }^{16}$ and they exhibit ferroelectric behavior in samples as thin as $1 \mathrm{~nm} .{ }^{17}$ The ferroelectric properties of the LB copolymer films - the phase-transition temperatures, the spontaneous polarization, and the piezoelectric and pyroelectric responses ${ }^{16,17}$ - are similar to those of films made by solvent spinning. ${ }^{2}$ The switching characteristics of the LB films, however, are fundamentally different. They have much higher coercive fields, ${ }^{18}$ about $500 \mathrm{MV} / \mathrm{m}$ vs $50 \mathrm{MV} / \mathrm{m}$ for solvent-formed films, ${ }^{19}$ and switching is much slower, ${ }^{20}$ more than $10 \mathrm{~s}$ vs less than $1 \mu \mathrm{s} .{ }^{21}$ The present study reveals some of the effects of electron irradiation on the properties of the LB films, particularly on the crystallinity, transition temperature, polarization, and switching characteristics.

\section{EXPERIMENTAL METHODS}

The samples consisted of capacitors containing LB films of $\mathrm{P}(\mathrm{VDF}-\mathrm{TrFE}$ 70:30) copolymer. The substrates were 0.15-mm-thick glass slides on which two 50-nm-thick, 1$\mathrm{mm}$-wide strip aluminum electrodes were evaporated. The 70:30 copolymer was deposited to the desired thickness by a method based on LB deposition, as described in detail elsewhere. ${ }^{15,22,23}$ Briefly, in this method, a $0.1 \mathrm{wt} \%$ copolymer solution in dimethylsulfoxide (DMSO) is dispersed onto the surface of a trough of water at room temperature. Barriers on the water's surface slowly compress the copolymer to a surface pressure of $5 \mathrm{mN} / \mathrm{m}$, which is well below the collapse pressure. At this pressure, we have found that the deposited LB films were uniform and highly oriented. After deposition, another set of identical top aluminum strip electrodes oriented $90^{\circ}$ with respect to the bottom electrodes were evaporated, allowing four independently addressable capacitors per sample. Twenty-four samples were used in this study: three 10-layer samples (18 $\mathrm{nm}$ thick), eighteen 20 layer samples $(36 \mathrm{~nm})$, and three 50-layer $(90 \mathrm{~nm})$ samples. The samples were annealed at $120{ }^{\circ} \mathrm{C}$ for $2 \mathrm{~h}$ prior to irradiation to improve crystallinity, resulting in uniform films with high crystallinity and orientation. Each sample was characterized prior to irradiation by measuring the thermal hysteresis, the coercive voltage, and the remanent polarization. The sample capacitance, which is proportional to the dielectric constant, was measured using an impedance analyzer (Hewlett-Packard $4192 \mathrm{~A})^{24}$ at $1-\mathrm{kHz}$ frequency and $0.1-\mathrm{V}$ amplitude. The capacitance was recorded as a function of temperature during heating and cooling at a rate of $1{ }^{\circ} \mathrm{C} / \mathrm{min}$ to characterize the ferroelectric-paraelectric and paraelectric-ferroelectric phase transitions, respectively. The capacitance was recorded as a function of the voltage bias cycled at a rate of $0.05 \mathrm{~V} / \mathrm{s}$ to characterize polarization switching.
The remanent polarization and switching characteristics were measured by the Merz and Chynoweth methods, respectively. ${ }^{25,26}$ The switched charge was determined by the Merz method, in which a sinusoidal or triangular voltage wave form was applied to a sample and the resulting current through the sample was measured. The current due to polarization switching appeared as a peak in excess of the usual capacitor discharge current, and the area of this peak equals twice the switched polarization. The Merz measurements were performed by applying triangular wave forms with amplitudes from 12 to $18 \mathrm{~V}$ at each of three different frequencies $(0.1,1$, and $100 \mathrm{~Hz})$ and recording the voltage across a fixed resistor connected in series with the sample. The Merz measurements were made both before and after irradiation. The Chynoweth method allows one to measure the pyroelectric response, which is proportional to the net sample polarization, by measuring the polarization current due to a small laser-induced thermal modulation. The Chynoweth pyroelectric measurements were made with a $5-\mathrm{mW}$ He-Ne laser chopped at $2 \mathrm{kHz}$ and the resulting ac was recorded by a digital lock-in amplifier (Stanford Research Systems SR830). To record the polarization hysteresis loops, an electrical bias voltage was applied to the sample for $1 \mathrm{~min}$, then the bias was removed and the sample was connected to the current inputs of the lock-in amplifier. The sample was illuminated with the modulated laser beam and the resulting ac current was measured at zero voltage by the lock-in amplifier and was allowed to stabilize for $5 \mathrm{~min}$. This was repeated as the applied voltage was cycled stepwise. The polarization retention measurements also used this same method, except that the polarization was saturated by applying a steady voltage of either $\pm 20 \mathrm{~V}$ for $1 \mathrm{~h}$ and then the pyroelectric current was measured continuously for up to $36 \mathrm{~h}$. The Chynoweth pyroelectric measurements were made only after irradiation.

Film crystallinity was measured by $\mathrm{x}$-ray diffraction using a Rigaku $\theta-2 \theta$ diffractometer with $\mathrm{Cu} K \alpha(1.54 \AA)$ radiation to measure the lattice spacing perpendicular to the film.

\section{IRRADIATION}

Irradiation of the samples was performed at the National Institute of Standards and Technology using a Van de Graaff electron accelerator. The samples were arranged in three identical vertical stacks of eight samples each and irradiated in a nitrogen atmosphere at room temperature. In this configuration, both exposure and electron energy will decrease as the beam passes through the stack. The beam area was $7.62 \times 7.62 \mathrm{~cm}^{2}$, which was large enough to simultaneously irradiate all three sample stacks with $\pm 5 \%$ lateral beam uniformity. This configuration allowed for irradiation of a large number of samples with a broad range of doses, from 0.16 to $1.10 \mathrm{MGy}$, to be obtained under uniform conditions. Because of the sample configuration, doses were only measured directly for the top samples, then calculated for lower samples by the method described below.

The sample chamber was mounted with the substrates oriented horizontally in vertical stacks $28 \mathrm{~cm}$ below the Van de Graaff exit window, so that the electron beam was inci- 
TABLE I. Total electron dose for each irradiated sample for the three sample stacks. The electron beam strikes sample 1 first.

\begin{tabular}{ccccc}
\hline \hline Position & $\begin{array}{c}\text { Thickness } \\
\text { (layers) }\end{array}$ & $\begin{array}{c}\text { Stack I dose } \\
(\mathrm{MGy})\end{array}$ & $\begin{array}{c}\text { Stack II dose } \\
(\mathrm{MGy})\end{array}$ & $\begin{array}{c}\text { Stack III dose } \\
(\mathrm{MGy})\end{array}$ \\
\hline 1 & 10 & $0.500 \pm 0.027$ & $0.750 \pm 0.040$ & $1.000 \pm 0.054$ \\
2 & 20 & $0.552 \pm 0.030$ & $0.828 \pm 0.045$ & $1.104 \pm 0.059$ \\
3 & 50 & $0.543 \pm 0.029$ & $0.814 \pm 0.044$ & $1.085 \pm 0.058$ \\
4 & 20 & $0.522 \pm 0.028$ & $0.783 \pm 0.042$ & $1.044 \pm 0.056$ \\
5 & 20 & $0.449 \pm 0.024$ & $0.674 \pm 0.036$ & $0.898 \pm 0.048$ \\
6 & 20 & $0.350 \pm 0.019$ & $0.525 \pm 0.028$ & $0.700 \pm 0.038$ \\
7 & 20 & $0.274 \pm 0.015$ & $0.410 \pm 0.022$ & $0.548 \pm 0.030$ \\
8 & 20 & $0.164 \pm 0.009$ & $0.246 \pm 0.013$ & $0.328 \pm 0.018$ \\
\hline \hline
\end{tabular}

dent vertically from above. In order to achieve lateral uniformity across the sample area, a $250-\mu \mathrm{m}$-thick aluminum scattering foil was placed in the beam $5 \mathrm{~cm}$ from the exit window. A primary electron-beam energy of $1.26 \mathrm{MeV}$ was used for the irradiations, with typical beam currents of about $75 \mu \mathrm{A}$. After passing through the intervening layers, the electron beam has an energy of $1 \mathrm{MeV} \pm 2 \%$ as it enters the sample stack. At this energy, a reasonable dose gradient was achieved while still giving a significant dose to the samples at the bottom of the stacks. All three stacks were present at the beginning of exposure and were removed one at a time as each received the desired dose. The stack ordering and doses are given in Table I.

\section{SAMPLE DOSE DETERMINATION}

Doses of the samples were determined using a combination of experimental and computational techniques. The experimental dose of the top sample was measured using radiochromic dye dosimeters, $1.0-\mathrm{cm}$ square and $50 \mu \mathrm{m}$ thick. ${ }^{27}$ These dosimeters consist of a nylon matrix incorporating an organic dye in the form of a thin solid film. They are well characterized, yield reproducible results, and come in a convenient form for measuring sample doses in the range of 1-50 kGy. Rather than placing a dosimeter film at each sample layer, it was decided to measure the dose of the top sample and use computational modeling to determine the doses of subsequent layers. This was done for two reasons: (1) A stack of eight dosimeters, one for each sample layer, would require the beam to pass through an additional $0.4 \mathrm{~mm}$ of the material, causing a significant beam attenuation. (2) Since the required sample doses exceeded the range of the dosimeter, the irradiation would have to be stopped to remove all eight dosimeters from the sample chamber before continuing. In order to properly relate the dose in the dosimeter to the total dose in the top sample, a method of charge normalization was used. Charge normalization was accomplished by means of a copper ring surrounding the chamber opening and hooked electrically to a current digitizer. In this way, the dose of the film could be related to the total charge collected in the ring. From this normalization factor the prescribed dose of the top sample could be obtained by irradiation until the proper charge value was reached.

Doses of the samples lower in the stacks were derived using the Integrated Tiger Series (ITS) Monte Carlo code. ${ }^{28}$

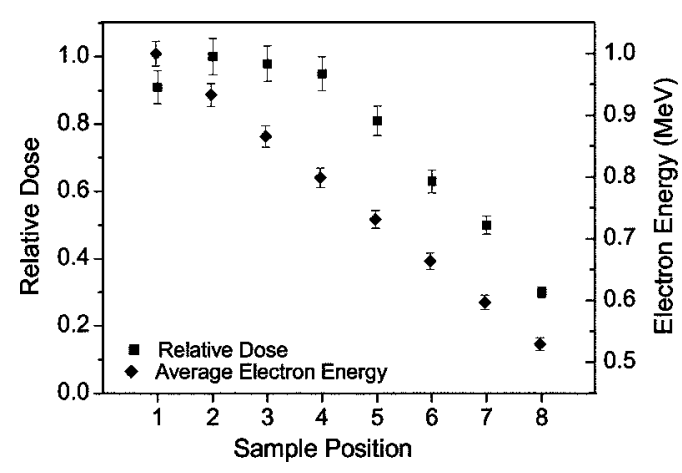

FIG. 1. Calculated relative dose and beam energy as a function of sample position. The electron beam strikes sample 1 first in each stack.

The code was used to generate a profile of the relative dose as a function of the sample depth using a detailed model of the experimental conditions, which accounted for all the layers in the beam path-foil, aluminum electrodes, polymer LB films, and substrates. This depth-dose profile was then used to obtain the dose of the other samples by normalizing to the measured dose in sample 1. A graph of the relative dose and beam energy at each sample position is given in Fig. 1. Note that the first sample does not receive the maximum dose, because even as fluence decreases as the beam penetrates the stack, the beam energy decreases and deposits proportionally higher doses. The main contributions to dose error are due to the dose calibration and the dye dosimeters. The beam energy was calculated using the Bethe-Bloch formula for the average energy loss in a material. ${ }^{29}$

\section{RESULTS}

Figure 2 shows the thermal hysteresis in the capacitance for three 20-layer samples that received different doses. The dielectric peaks on heating and cooling correspond to the ferroelectric-paraelectric and converse transitions, respectively. ${ }^{1}$ Irradiation results in a monotonic downward shift for both transition peaks, as shown in Fig. 3, at a rate of $-46{ }^{\circ} \mathrm{C} / \mathrm{MGy}$ for the heating peaks and $-57{ }^{\circ} \mathrm{C} / \mathrm{MGy}$ for the cooling peaks up to a dose of $0.60 \mathrm{MGy}$. These transition

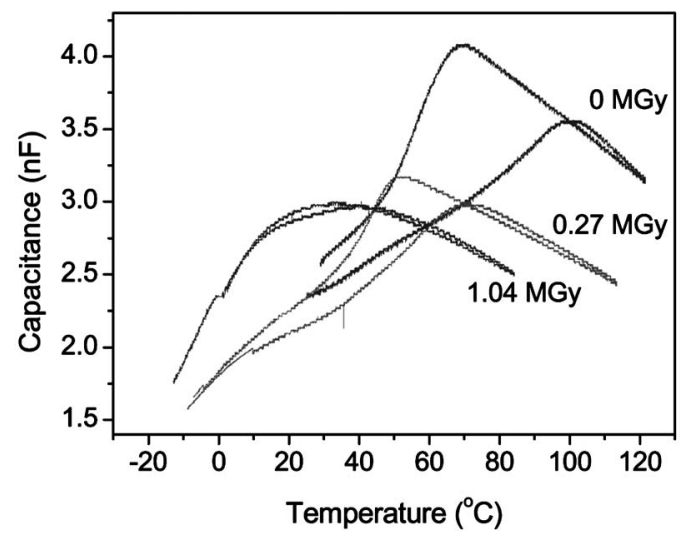

FIG. 2. Capacitance measurements showing thermal hysteresis for three of the 20-layer samples receiving different electron-beam doses, as labeled next to the curves. Notice the downward shift in the heating and cooling phase-transition peaks and the virtual elimination of thermal hysteresis accompanied by peak broadening at 1.04-MGy dose. 

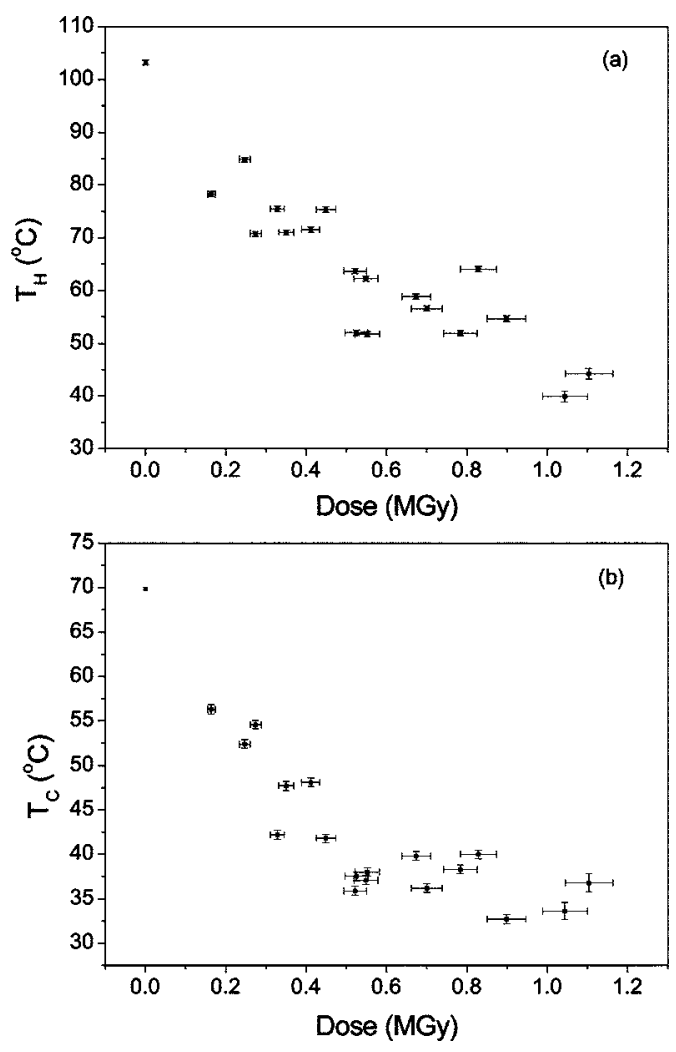

FIG. 3. The dielectric peak temperature (from Fig. 2) as a function of dose for heating (a) and cooling (b) for the 20-layer samples. The slope is $-46{ }^{\circ} \mathrm{C} / \mathrm{MGy}$ on heating and $-57^{\circ} \mathrm{C} / \mathrm{MGy}$ on cooling up to $0.60 \mathrm{MGy}$ where values appear to plateau.

temperature suppression rates are comparable to those obtained from studies of thicker spun-cast and hot-pressed VDF copolymer films. ${ }^{7,11,12}$ There is a significant thermal hysteresis evident for 20-layer samples receiving doses up to $0.90 \mathrm{MGy}$, and up to $1.08 \mathrm{MGy}$ for the 50-layer samples. Higher doses eliminate thermal hysteresis. The suppression may begin to plateau, as seen by Cheng et al., ${ }^{11}$ for doses higher than 0.70 MGy. However, this issue is confounded by the increase in the fraction of the amorphous material, which is evident in the broadening of the phase-transition peak for high doses (see Fig. 2 for 1.04-MGy dose). The dielectric peak for 1.04 MGy may no longer be due to a true ferroelectric-paraelectric phase transition, but may result from a dielectric anomaly in the amorphous regions. Both Lovinger $^{12}$ and Daudin et al. ${ }^{7}$ reported a linear decrease of $T_{C}$ with increased electron dose. Lovinger's differential scanning calorimetry (DSC) data, in particular, shows no discernible phase transition above 1.0-MGy dose using 3-MeV electrons.

Figure 4 shows the capacitance, $C(E) \propto d D / d E$, "butterfly" curves for three 20-monolayer samples receiving different electron doses. The butterfly curves show peaks near the coercive voltage, the voltage at which the spontaneous polarization reverses direction. ${ }^{30}$ The coercive voltage is shown to decrease with increased dose at a rate of $-2.2 \mathrm{~V} / \mathrm{MGy}$, as shown in Fig. 5. The amount of hysteresis in the butterfly curves also decreases with increased dose, which is likely due to a decrease in the remanent polarization. The values of capacitance do not show a clear dependence on dose, and

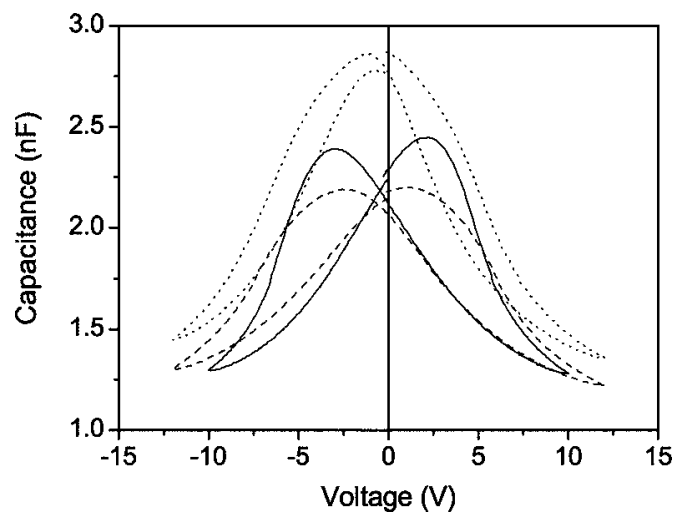

FIG. 4. Capacitance butterfly curves for three 20-layer samples of different doses: 0 MGy (solid), 0.27 MGy (dashed), and 1.04 MGy (dotted).

any differences seen are likely due to sample variation. Just like the thermal dielectric peak, this measurement is also sensitive to the dielectric response of the amorphous material, and therefore the peak voltage given by this measurement may not correspond to the true coercive voltage of the ferroelectric material, especially for higher doses.

The dielectric measurements can be influenced strongly by a relaxorlike behavior, as has been shown by Zhang and co-workers, ${ }^{9-11}$ and therefore the coercive voltage and the remanent polarization were independently measured using the Chynoweth pyroelectric technique. ${ }^{26}$ The pyroelectric current is directly proportional to the net polarization in the film, ${ }^{31}$ and probes only the ferroelectric phase unlike the capacitance, which includes contributions from all crystalline and amorphous phases. Figure 6(a) shows the pyroelectric hysteresis loops for samples of different doses. The coercive voltage was determined from the pyroelectric hysteresis loops by taking half of the difference between the intercept voltages. The coercive voltages shown in Fig. 5 indicate that the switching characteristics of the films have been affected by irradiation. The butterfly capacitance measurements include contributions from amorphous and crystalline materials of any phase, not just the ferroelectric material. The pyroelectric measurements are sensitive to only the ferroelectric crystalline material, but the present data cannot determine whether the changes are due to the presence of

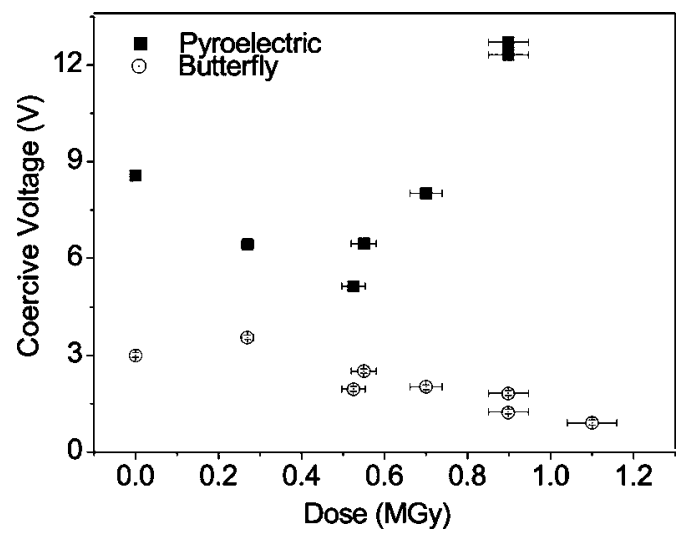

FIG. 5. Coercive voltage determined from the butterfly capacitance peaks (Fig. 4) and the pyroelectric hysteresis loops [Fig. 6(a)] for the 20-layer samples. 

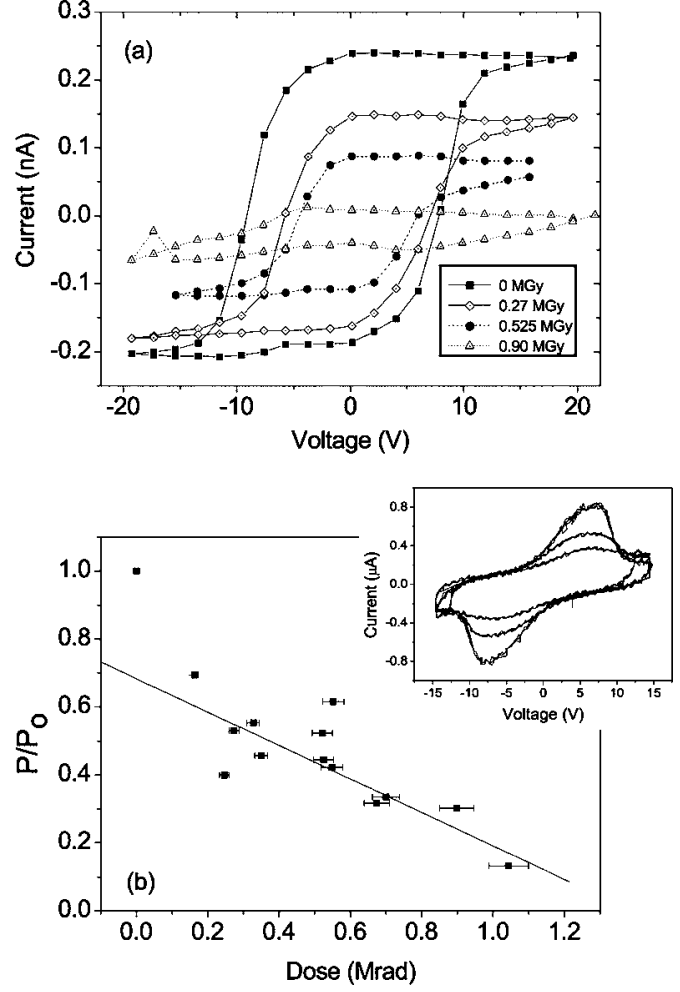

FIG. 6. (a) Pyroelectric hysteresis loops of samples receiving different doses. (b) Ratio of the remanent polarization after irradiation $(P)$ to that before irradiation $\left(P_{o}\right)$ as a function of dose. Inset: Merz switching current loops for samples receiving 0-, 0.27-, and 0.90-MGy doses.

increased amorphous material, the reduction of ferroelectric crystal size, increased strain, or to an interaction between the amorphous and crystalline regions. The saturated (maximum) pyroelectric signal is found to decrease monotonically with increasing dose at a rate of $-83 \% / \mathrm{MGy}$, as shown in Fig. 7. Polarization values obtained from the Merz data [Fig. 6(b) ] revealed a similar reduction in the polarization, but a lower rate of $-60 \% / \mathrm{MGy}$. The Merz measurements are dynamic measurements made at $1 \mathrm{~Hz}$, cycled much faster than the butterfly or pyroelectric measurements, and may not include the total switched charge due to dispersion in the switching times across the film. The presence of repeatable hysteresis indicates that a ferroelectric material is present in all the irradiated films up to at least $0.90 \mathrm{MGy}$, although the

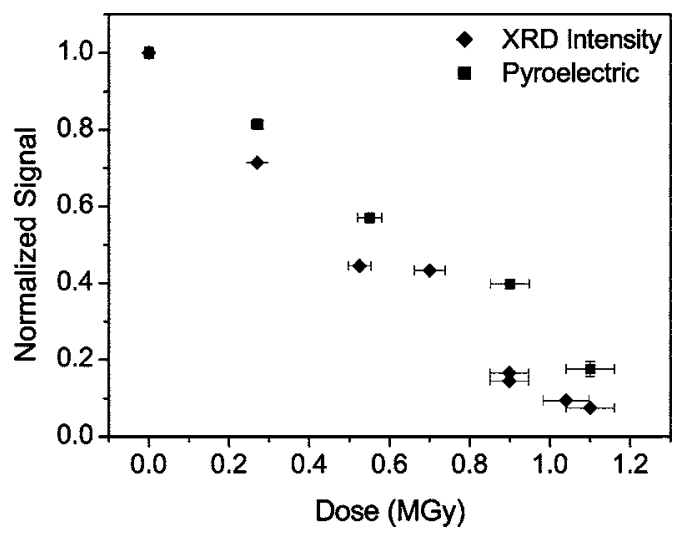

FIG. 7. Normalized $\mathrm{x}$-ray diffraction intensity and pyroelectric current as a function of electron dose for the 20-layer samples.
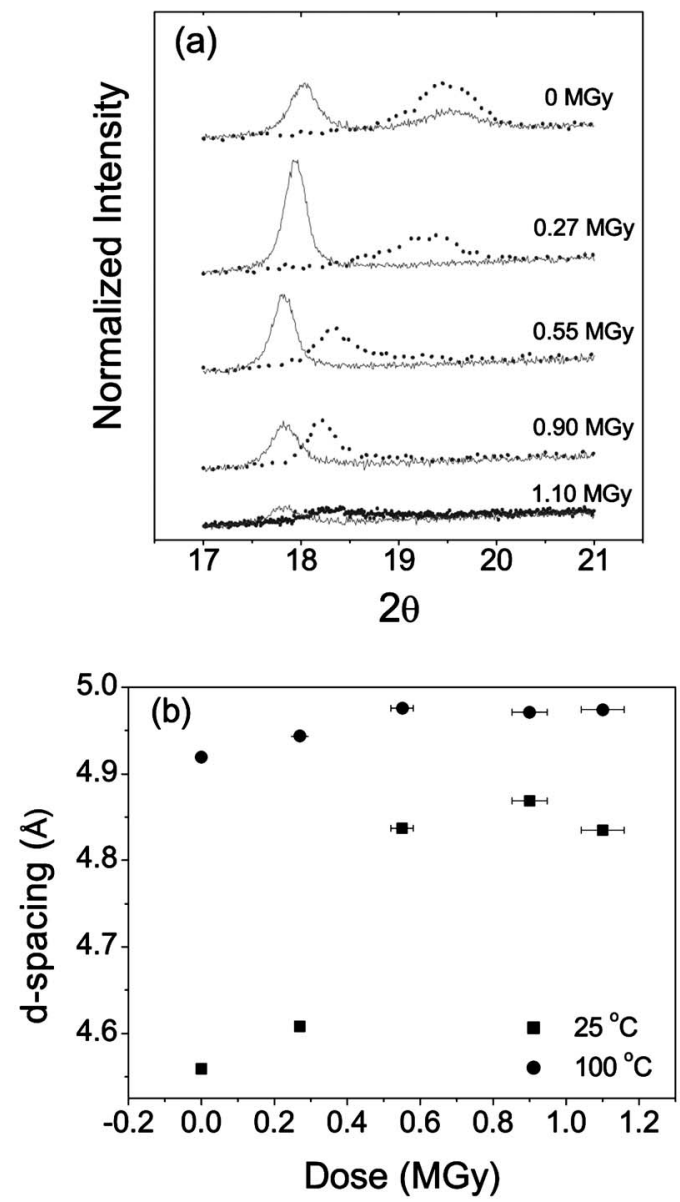

FIG. 8. (a) X-ray diffraction data showing the (110) peaks from the 20-layer samples of different doses at room temperature (dotted line) and at $100{ }^{\circ} \mathrm{C}$ (solid line). (b) (110) diffraction peak $d$ spacings vs dose at room temperature (squares) and at $100{ }^{\circ} \mathrm{C}$ (circles).

remanent polarization of the samples was greatly reduced. Furthermore, samples receiving up to 0.90-MGy dose maintained stable polarization for at least $36 \mathrm{~h}$, after an initial decay of $30 \%-50 \%$ in the first $5 \mathrm{~min}$. This initial decrease may indicate the presence of trapped charge or crystallites with unstable polarization.

The $\theta-2 \theta$ x-ray diffraction measurements show the (110) peak normal to the film. For the ferroelectric phase, the (110) peak is located near $19.5^{\circ}$, corresponding to a layer spacing of $4.5 \AA$, while the (110) paraelectric phase peak is located near $18^{\circ}$, corresponding to a spacing of $4.9 \AA .^{16,32}$ Figure 8(a) shows the $\mathrm{x}$-ray diffraction measurements recorded at room temperature and at $100{ }^{\circ} \mathrm{C}$ for the 20-layer films of different doses. At $100{ }^{\circ} \mathrm{C}$, the unirradiated sample shows both ferroelectric and paraelectric peaks, indicating that the sample is still in a mixed phase, which is consistent with the fact that its dielectric peak on heating is at $100{ }^{\circ} \mathrm{C}$ (see Fig. $3)$. The irradiated samples exhibit only the paraelectric X-ray diffraction (XRD) peak at $100{ }^{\circ} \mathrm{C}$, because their transition temperatures have been suppressed, and no ferroelectric phase remains at this temperature. The room-temperature measurements show the gradual conversion of the sample from the ferroelectric phase to the paraelectric phase. Figure 8(b) shows the (110) $d$ spacings of the films plotted as a function of dose. The ferroelectric $\beta$-phase peak decreases to 
zero at approximately $0.55 \mathrm{MGy}$ and is partially replaced by a spacing near the paraelectric phase spacing. The total integrated intensity of the ferroelectric and paraelectric peaks decreases to zero at approximately 1.20 MGy, which implies that the material is almost completely amorphous, though it does not rule out the presence of crystallites with a different orientation. The presence of the paraelectric phase at room temperature is in contrast with the thermal hysteresis found in the capacitance versus temperature measurements and polarization hysteresis measurements, which would indicate that there is a substantial ferroelectric material at room temperature, even up to 0.90-MGy exposure. It has been suggested that at room temperature, the ferroelectric regions are too small to be seen in x-ray diffraction, which places a maximum size of $5 \mathrm{~nm}$ on the ferroelectric crystallites at $0.50 \mathrm{MGy} .{ }^{11}$ This seems unlikely in this case, however, if one considers the correlation between the crystallinity and remanent polarization on irradiation dose, as shown in Fig. 7. The crystallinity decreases at a rate of $-72 \% / \mathrm{MGy}$, while the net pyroelectric signal decreases at a rate of $-85 \% / \mathrm{MGy}$. This linear decrease in polarization was also observed in the total switched charge calculated from the Merz measurements, as shown in Fig. 6(b). The correlation between polarization and crystallinity indicates that most or all of the remaining crystalline material is also polarizable under an electric field, and hence ferroelectric. Previous studies have shown a large reconversion of paraelectric material to ferroelectric material under an electric field, which may be partially responsible for the giant electrostriction following electron irradiation of these materials. ${ }^{11}$ However, the small area of our electrodes makes this observation difficult to quantify because the area of our x-ray beam is about 100 times larger than our electrode area.

\section{CONCLUSIONS}

The present results indicate that irradiation mainly reduces sample crystallinity. The remaining ferroelectric material has a slightly reduced transition temperature, though a combination of thermal annealing and poling is sufficient to return the crystalline fraction to the ferroelectric phase at room temperature. The strong dielectric nonlinearity and hysteresis evident in the butterfly curves of the highly irradiated samples suggest that the amorphous material is primarily responsible for a relaxorlike behavior observed with solvent-formed copolymer films, which exhibit large electrostriction after doses in the range from 0.6 to $0.8 \mathrm{MGy} .{ }^{9,11}$ A measurement of the electrostriction in the irradiated LB films is needed to test this hypothesis.

Future studies should focus on determining the types and amounts of defects acquired during irradiation and on the effect of irradiation on electrostriction and switching kinetics. Electrostriction measurements correlated with crystallinity and remanent polarization are necessary to confirm the assumption that phase conversion is the primary cause of large electrostriction in $\mathrm{P}(\mathrm{VDF}-\mathrm{TrFE})$. Identifying the types of defects will give insight to the reduction in the phasetransition temperature and the relaxation of the crystal struc- ture. This identification may also help identify the cause of the increase in coercive field above 0.5 MGy and aid in determining whether these defects impede or enhance the switching characteristics of the LB films.

\section{ACKNOWLEDGMENTS}

This work was supported by the National Science Foundation and the Nebraska Research Initiative.

${ }^{1}$ The Applications of Ferroelectric Polymers, edited by T. T. Wang, J. M. Herbert, and A. M. Glass (Chapman and Hall, New York, 1988).

${ }^{2}$ T. Furukawa, Phase Transitions 18, 143 (1989).

${ }^{3}$ A. J. Lovinger, Science 220, 1115 (1983).

${ }^{4}$ A. J. Lovinger, in Developments in Crystalline Polymers, edited by D. C. Basset (Applied Science, London, 1982), Vol.1.

${ }^{5}$ A. J. Lovinger, G. T. Davis, T. Furukawa, and M. G. Broadhurst, Macromolecules 15, 323 (1982).

${ }^{6}$ F. Macchi, B. Daudin, and J. F. Legrand, Ferroelectrics 109, 303 (1990).

${ }^{7}$ B. Daudin, J. F. Legrand, and F. Macchi, J. Appl. Phys. 70, 4037 (1991).

${ }^{8}$ V. Bharti, H. Xu, G. Shanthi, Q. M. Zhang, and K. Liang, J. Appl. Phys. 87, 452 (2000).

${ }^{9}$ V. Bharti, G. Shanthi, H. Xu, Q. M. Zhang, and K. Liang, Mater. Lett. 47, 107 (2001)

${ }^{10}$ Q. M. Zhang, V. Bharti, and X. Zhao, Science 280, 2101 (1998).

${ }^{11}$ Z.-Y. Cheng, Q. M. Zhang, and F. B. Bateman, J. Appl. Phys. 92, 6749 (2002).

${ }^{12}$ A. J. Lovinger, in Radiation Effects on Polymers, edited by R. L. Clough and W. Shalaby (American Chemical Society, Washington, DC, 1991), Chap. 6.

${ }^{13}$ A. J. Lovinger, Macromolecules 18, 910 (1985).

${ }^{14}$ S. T. Lau, K. Y. Leung, H. L. W. Chan, C. L. Choy, B. Sundaravel, and I. Wilson, Ferroelectrics 273, 9 (2002).

${ }^{15}$ S. Palto, L. Blinov, A. Bune, E. Dubovik, V. Fridkin, N. Petukhova, K. Verkhovskaya, and S. Yudin, Ferroelectr., Lett. Sect. 19, 65 (1995).

${ }^{16}$ J. Choi et al., Phys. Rev. B 61, 5760 (2000).

${ }^{17}$ A. V. Bune, V. M. Fridkin, S. Ducharme, L. M. Blinov, S. P. Palto, A. V. Sorokin, S. G. Yudin, and A. Zlatkin, Nature (London) 391, 874 (1998).

${ }^{18}$ S. Ducharme, V. M. Fridkin, A. V. Bune, S. P. Palto, L. M. Blinov, N. N. Petukhova, and S. G. Yudin, Phys. Rev. Lett. 84, 175 (2000).

${ }^{19}$ K. Kimura and H. Ohigashi, Appl. Phys. Lett. 43, 843 (1983).

${ }^{20}$ G. Vizdrik, S. Ducharme, V. M. Fridkin, and S. G. Yudin, Phys. Rev. B 68, 094113 (2003).

${ }^{21}$ T. Furukawa, M. Date, M. Ohuchi, and A. Chiba, J. Appl. Phys. 56, 1481 (1984).

${ }^{22}$ S. Ducharme, S. P. Palto, and V. M. Fridkin, in Handbook of Thin Film Materials, edited by H. S. Nalwa (Academic, San Diego, 2002), Vol. 3, Chap. 11.

${ }^{23}$ A. Sorokin, S. Palto, L. Blinov, V. M. Fridkin, and S. Yudin, Mol. Mater. 6, 61 (1996).

${ }^{24}$ Certain commercial equipment, instruments, or materials are identified in this paper to specify the experimental procedure adequately. Such identification is not intended to imply recommendation or endorsement by the National Institute of Standards and Technology, nor is it intended to imply that the materials or equipment identified are necessarily the best available for the purpose.

${ }^{25}$ W. J. Merz, J. Appl. Phys. 27, 938 (1956).

${ }^{26}$ A. G. Chynoweth, J. Appl. Phys. 27, 78 (1956).

${ }^{27}$ J. C. Humphreys, D. Hocken, and W. L. McLaughlin, NBS Measurement Services: Dosimetry for High-Dose Applications (National Bureau of Standards, Gaithersburg, MD, 1988), publication number 250-11.

${ }^{28}$ J. A. Halbleib, R. P. Kensek, T. A. Mehlhorn, G. D. Valdez, S. M. Seltzer, and M. J. Berger, Sandia National Laboratories Report No. SAND911634, 1994.

${ }^{29}$ R. M. Sternheimer, S. M. Seltzer, and M. J. Berger, Phys. Rev. B 26, 6067 (1982).

${ }^{30}$ S. Ducharme, A. V. Bune, L. M. Blinov, V. M. Fridkin, S. P. Palto, A. V. Sorokin, and S. G. Yudin, Phys. Rev. B 57, 25 (1998).

${ }^{31}$ A. V. Bune, C. Zhu, S. Ducharme, L. M. Blinov, V. M. Fridkin, S. P. Palto, N. N. Petukhova, and S. G. Yudin, J. Appl. Phys. 85, 7869 (1999).

${ }^{32}$ E. Bellet-Amalric and J. F. Legrand, Eur. Phys. J. B 3, 225 (1998). 